\title{
Estrategias para combatir el edadismo: ¿formación específica sobre envejecimiento 0 contacto intergeneracional?
}

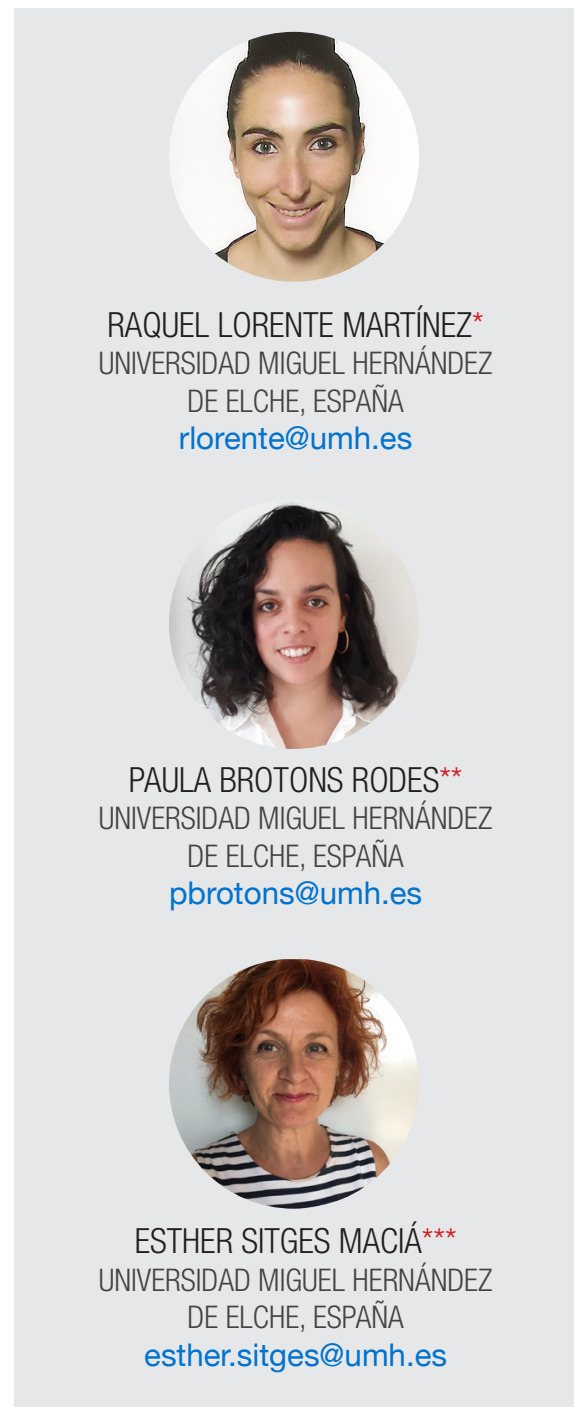

\section{Strategies to combat ageism: positive education about aging or intergenerational contact?}

Recibido: 25 de septiembre de 2019 | Aprobado: 18 de noviembre de 2019

\section{Resumen}

Desde el Modelo PEACE se sugiere que realizar intervenciones combinadas (educación sobre el envejecimiento y contacto intergeneracional) es la mejor estrategia para combatir el edadismo. Este estudio pretende observar el impacto del aprendizaje experiencial al comprobar empíricamente si el contacto intergeneracional tiene efectos sobre los estereotipos negativos hacia la vejez de un grupo de estudiantes que había recibido previamente formación teórica sobre el envejecimiento en un programa de voluntariado universitario. Se realizó un diseño pre-post sin grupo control con 18 participantes del programa. Los estereotipos negativos hacia la vejez se evaluaron mediante la escala CENVE, comparando los datos de los participantes en dos momentos temporales (tras recibir la formación teórica y tras realizar la práctica de contacto intergeneracional). Se observó una reducción estadísticamente significativa de los estereotipos relacionados con aspectos motivacionales y sociales del envejecimiento tras realizar la práctica de contacto intergeneracional, aspecto en el que no mostraron cambios tras haber recibido la formación teórica del Programa, respecto al pre-test. Este hallazgo evidencia el potencial de los programas combinados para eliminar estereotipos edadistas en contextos educativos.

Palabras clave: estereotipos, edadismo, vejez, educación, aprendizaje experiencial.

Profesora Asociada del Departamento de Psicología de la Salud y Coordinadora de Voluntariado de SABIEX de la Universidad Miguel Hernández de Elche, España. Para contactar a la autora: rlorente@umh.es

** Psicóloga especialista en Gerontología y Salud y colaboradora de SABIEX de la Universidad Miguel Hernández de Elche, España. Para contactar a la autora: pbrotons@umh.es

*** Profesora Titular del Departamento de Psicología de la Salud y Directora del Programa de Mayores SABIEX de la Universidad Miguel Hernández de Elche, España. Para contactar a la autora: esther.sitges@umh.es; Teléfono: +34966658528 


\section{Abstract}

From the PEACE Model, it is suggested that carrying out combined interventions (education on aging and intergenerational contact) is the best strategy to combat ageism. This study aims to observe the effects of experiential learning by empirically checking whether intergenerational contact has an effect on negative stereotypes towards old age of a group of students who had previously received theoretical training on aging in a university volunteer program. A pre-post design was carried out without a control group with 18 participants. Negative stereotypes towards old age were evaluated using the CENVE scale at two times (after receiving the theoretical training and after performing the practice of intergenerational contact). A statistically significant reduction of stereotypes related to motivational and social aspects of aging was observed after the practice of intergenerational contact, an aspect in which they showed no changes after receiving the theoretical training of the Program, with respect to the pre-test. This finding demonstrates the potential of combined programs to eliminate ageism stereotypes in educational contexts.

Keywords: stereotypes, ageism, old age, education, experiential learning.

\section{Introducción}

La visión que la sociedad tiene de las personas mayores es principalmente negativa y centrada en una imagen mitificada de declive físico (aumento de la incidencia de enfermedades, declive de las capacidades funcionales, etc.), motivacional (falta de interés), social (desvinculación social y soledad) y de personalidad (rigidez e inflexibilidad, poca creatividad, etc.) (Moreno, 2010; Carbajo-Vélez, 2009). Esta visión estereotipada de la vejez ha sido definida como edadismo/ageism (Butler, 1969) y se refiere al conjunto de estereotipos y prejuicios asociados a las personas mayores y al proceso de envejecer que compromete el envejecimiento activo y saludable.

El edadismo se ve reforzado y sobregeneralizado a través de las representaciones sociales que se trasmiten en medios de comunicación manifestando continuamente, de forma arraigada, esta percepción mitificada de la vejez como colectivo minoritario, centrado en el temor al deterioro y a la pérdida de autonomía. Además, en los anuncios que se emiten desde estos medios se fomenta una visión pasiva de la vejez, clasificando al colectivo de personas mayores dentro del grupo de consumidores y no como productores (del Campo y Maestro, 2014).

Esta visión negativa del envejecimiento puede influir en la percepción que las personas mayores tienen de sí mismas, mediante el efecto Pigmalión o profecía autocumplida, favoreciendo que se ajusten a la imagen negativa que la sociedad tiene sobre ellas; comprometiendo así la promoción del envejecimiento activo y saludable en esta población (Menéndez, Cuevas-Toro, Pérez-Padilla y Lorence, 2016).

La Organización Mundial de la Salud (OMS) advierte que el aumento de la población mayor de 65 años a nivel mundial, que en España representa ya al 19.1\% de la población (Abellán-García et al., 2019), precisa que se aborde el envejecimiento de la población desde la salud pública, a través del desarrollo de nuevas políticas que eviten los preconceptos y estereotipos negativos asociados a las personas mayores $\mathrm{y}$, por consiguiente, la discriminación por razón de edad (OMS, 2015). Para eliminar esta visión estereotipada, es preciso transmitir a la sociedad una información sobre la vejez que proporcione una imagen más diversa y positiva del envejecimiento, favoreciendo así una reconceptualización de la idea de la vejez más ajustada a la realidad (Blanco-Molina y PinazoHernandis, 2016; del Campo y Maestro, 2014).

En este sentido, diversos estudios confirman que las generaciones más jóvenes tienen una percepción negativa hacia la vejez y señalan la necesidad de formar en materia de envejecimiento tanto a la población infantil como la juvenil (Gutiérrez y Mayordomo, 2019; Rello, Bravo, y Plata, 2018; Sarabia y Castanedo, 2015; Sanhueza-Chamorro, 2014). 
En la población universitaria, Gutiérrez y Mayordomo (2019) comprobaron que el alumnado de grado de Magisterio presentaba estereotipos negativos hacia la vejez, sobre todo, en aspectos de carácter y personalidad y motivación social, atribuyendo a las personas mayores una mayor rigidez mental y menos intereses vitales. Sanhueza-Chamorro (2014) observó que los estudiantes de Ciencias de la Salud tenían una imagen generalmente más positiva que los estudiantes de Ciencias Sociales. Asimismo, teniendo en cuenta los años de estudio y la práctica profesional en diferentes áreas de especialización sanitaria, Rello, Bravo y Plata (2018), comprobaron, con una muestra de 200 estudiantes de grado de Fisioterapia y Terapia Ocupacional y profesionales de ambas disciplinas, que los estudiantes de primer curso de grado tenían significativamente más estereotipos edadistas que el grupo de estudiantes de cuarto curso y el de profesionales. Además, por disciplinas se observó que el estudiantado de cuarto curso de Fisioterapia mostraba más estereotipos negativos que el grupo de fisioterapeutas, sin embargo, esa diferencia no se observó entre el estudiantado de último curso de Terapia Ocupacional y el grupo de profesionales de esa especialidad. Los resultados de ese estudio demuestran que recibir una formación más específica en una disciplina dentro de las Ciencias de la Salud influye positivamente en la reducción de los estereotipos edadistas y, asimismo, que la experiencia práctica profesional con personas mayores también ayuda a mejorar la imagen sobre las personas mayores.

La investigación disponible hasta ahora sobre la efectividad de las intervenciones para combatir los estereotipos edadistas se puede clasificar fundamentalmente en dos categorías: una, centrada en la formación específica gerontológica basada en la trasmisión de conocimientos actualizados sobre la heterogeneidad del proceso de envejecer; y otra, centrada en el contacto intergeneracional a través del desarrollo de experiencias de interacción social tanto individualizadas como en grupo.

Desde la primera aproximación nos encontramos con estudios que defienden que, a través de la formación especifica sobre aspectos gerontológicos, se puede desarrollar una reconceptualización del proceso de envejecimiento en el que este proceso vital se normalice (Gutiérrez y Mayordomo, 2019; Ribera, Bustillos, Guerra, Huici y Fernández-Ballesteros, 2016; Sarabia y Castanedo, 2015; Borrella, 2013). Al proporcionar formación específica, aumenta el conocimiento sobre la vejez y se atiende menos a los mitos socialmente extendidos, disminuyendo así los estereotipos y actitudes negativas hacia este colectivo (Lytle, 2016; Carbajo-Velez, 2009; Snyder, Wesley, Lin y May, 2008). De este modo, si las intervenciones se llevasen a cabo a edades tempranas, los menores verían a las personas mayores como individuos activos dentro de la sociedad, pero con actividades diferentes, como en cualquier otro momento del ciclo vital (Gutiérrez y Mayordomo, 2019).

Por otro lado, desde la aproximación que propone promover el contacto intergeneracional, PinazoHernandis y Pinazo-Clapés (2018) encontraron una disminución significativa en los estereotipos de los jóvenes tras interactuar con personas mayores en una intervención semestral con sesiones semanales en las que jóvenes y mayores institucionalizados comentaban obras literarias. En otro programa con población universitaria, Elliott y Rubio (2017) comprobaron que los estudiantes que participaron conversando con una persona mayor durante las nueve sesiones del programa, superaron sus estereotipos previos sobre el envejecimiento. Otro programa universitario, en el que jóvenes y mayores trabajan juntos para diseñar un proyecto de investigación, mostró que los jóvenes aumentaban la sensibilidad, la solidaridad intergeneracional y las habilidades comunicativas (Gonçalves, Hatton-Yeo y Farcas, 2016).

Ambas aproximaciones de intervención del edadismo se integran en el modelo de Levy (2016), denominado PEACE (Positive Education about Aging and Contact Experiences). Este modelo propone que los estereotipos edadistas pueden reducirse a través de dos factores que están interconectados: una vertiente educativa o de formación sobre aspectos realistas y más positivos de la vejez y el envejecimiento; y otra vertiente basada en las experiencias reales entre generaciones a través 
del contacto intergeneracional con una persona mayor, centrada en compartir las vivencias y experiencias individuales, favorecer un estatus igualitario y trabajar de forma cooperativa con un objetivo común. De este modo, la combinación entre educación y contacto, cuando es posible, parece una estrategia de reducción del edadismo más eficaz que cualquiera de las otras estrategias de forma independiente: la educación proporciona el conocimiento esencial acerca del envejecimiento y la tercera edad y el contacto intergeneracional permite la exposición real y directa con personas mayores que genera una experiencia de aprendizaje concreta y accesible.

Con el objetivo de poner este modelo a prueba, Lytle y Levy (2017), realizaron dos estudios experimentales, uno con estudiantes universitarios y otro en población general, en los que compararon la efectividad de tres tipos de intervención (una formativa basada en la transmisión de datos empíricos sobre el proceso de envejecimiento, otra formativa basada en la trasmisión de información sobre experiencias reales de contacto intergeneracional y una intervención formativa combinada) con un grupo control. Las autoras comprobaron que la reducción de los estereotipos era similar en los tres grupos de intervención. Al no encontrar diferencias estadísticamente significativas entre las intervenciones, concluyeron que ambas estrategias formativas son igualmente efectivas $y$, como la combinación de estas no daba mejores resultados, no era preciso utilizar una intervención combinada para combatir el edadismo.

No obstante, estas autoras también señalaron la posibilidad de aplicar una variante de intervención de contacto intergeneracional más extensa, que consistiría en sustituir la formación basada en la transmisión de experiencias de contacto intergeneracional por una intervención de contacto intergeneracional real, que cumpliese con las cinco condiciones óptimas para fomentar un contacto intergeneracional positivo indicadas por Levy (2016), esto es: centrada en compartir las vivencias y experiencias individuales; favorecer un estatus igualitario y trabajar de forma cooperativa con un objetivo común, lo que permitiría comparar el efecto de la formación y el contacto directo para reducir estereotipos edadistas. Sin embargo, las autoras, hasta donde se ha podido comprobar en la revisión de la literatura científica disponible, no han llegado a aplicar este diseño (Lytle y Levy, 2017) y no se conocen investigaciones que hayan tratado de aportar más claridad en este sentido.

Así pues, y desde este enfoque dual, se diseñó un programa de voluntariado universitario que combina una formación teórica sobre el envejecimiento y una formación práctica de acompañamiento a una persona mayor (Programa Acompaña-Té). Este Programa es una formación complementaria que se ofrece a la comunidad universitaria y se fundamenta en los principios del aprendizaje experiencial, promoviendo contextos de aprendizaje donde, además de aplicar técnicas y habilidades, puedan reflexionar sobre su conocimiento y aspectos éticos y sociales (Zabalza, 2013). En este caso, el enfoque del aprendizaje experiencial del Programa persigue conseguir un cambio en las actitudes de los jóvenes hacia las personas mayores a partir del aumento de la conciencia sobre las implicaciones del envejecimiento y la acción social con este colectivo. De este modo, y siguiendo los componentes del aprendizaje a partir de la experiencia propuestos por Zabalza (2013), los estudiantes que participaron en la parte práctica de acompañamiento, no solo tenían que realizar las acciones planificadas de acompañamiento (situación externa de aprendizaje), sino que también tenían que reflexionar cada día y al final de la experiencia sobre el acompañamiento realizado (proceso interno de reflexión).

Para comprobar la efectividad de la formación teórica en los estudiantes universitarios que participaron en dicho Programa, se realizó un estudio previo en el que se observó que los participantes redujeron los estereotipos negativos hacia la vejez en las dimensiones Salud y Carácter-personalidad del Cuestionario de Estereotipos Negativos hacia la Vejez (Brotons, Lorente y Sitges, en prensa). A partir de los resultados encontrados en dicho trabajo, este estudio pretende observar los efectos del aprendizaje experiencial y comprobar si existen diferencias significativas en los estereotipos negativos de los estudiantes que realizaron el acompañamiento 
de una persona mayor (intervención de contacto intergeneracional), respecto al nivel de estereotipos que presentaron al finalizar la formación teórica (intervención educativa) del curso de voluntariado del Programa Acompaña-Té.

El presente estudio, por tanto, se ha diseñado a partir de los últimos hallazgos teóricos y empíricos sobre el abordaje del edadismo en contextos educativos y supone una aportación novedosa a la literatura científica existente sobre este tema. Los hallazgos de esta investigación permitirán comprobar el valor añadido que puede tener el aprendizaje experiencial, a través del contacto intergeneracional, en la reducción de estereotipos negativos sobre la vejez. Del mismo modo, ayudará a aportar más evidencias empíricas al modelo PEACE propuesto por Levy (2016), al analizar la efectividad de dicha intervención en los mismos participantes que realizaron la intervención educativa del Programa Acompaña-Té.
A continuación, se describe el método y procedimiento seguido para estudiar los efectos del aprendizaje experiencial en los participantes del Programa. Seguidamente, se exponen los resultados obtenidos y se discuten los mismos con los resultados de estudios anteriores. Para finalizar, se concluye la efectividad de la estrategia educativa implementada y se realizan recomendaciones sobre su aplicación en un entorno de aprendizaje similar.

\section{Método}

Participantes

En este estudio participaron 18 personas pertenecientes a la comunidad universitaria, con edades comprendidas entre los 18 y 54 años ( $M$ = 22.89; $D T=9.93$ ). Del total de los integrantes, el $83.3 \%$ mujeres y el $16.7 \%$ hombres. Además, el 33.3\% tenía experiencia previa de voluntariado.

Tabla 1. Datos sociodemográficos de los participantes

Frecuencia $\quad \%$

Nacionalidad

Española

Marroquí

Titulación
Periodismo

Psicología

Comunicación Audiovisual

Terapia Ocupacional

Ciencias Políticas

Trabajo Social
17

1

$94.4 \%$

$5.6 \%$

$22.2 \%$

$50 \%$

$1 \quad 5.6 \%$

2

$11.1 \%$

$1 \quad 5.6 \%$

$1 \quad 5.6 \%$ 


\section{Medidas}

Se aplicó una de las escalas más utilizadas en población hispanohablante para evaluar las creencias estereotipadas hacia la vejez, el Cuestionario de Evaluación de Estereotipos Negativos hacia la Vejez (CENVE) validado en población española joven y adulta por Menéndez et al. (2016). Es una escala compuesta por 15 ítems escritos de forma directa, con una escala de respuesta tipo Likert de cuatro alternativas de respuesta, cuyas opciones fluctúan desde $<<$ Totalmente en desacuerdo $>>$ hasta $<<$ Totalmente de acuerdo $>>$. A su vez, la escala se subdivide en tres dimensiones: Salud, Motivacional-social, Carácter-personalidad. De esta forma, el instrumento ofrece una puntuación total que oscila de 15 a 60 puntos indicando el grado de estereotipos negativos hacia la vejez (15-28 muy bajo, 29-39 bajo, 40-50 alto y 51-60 muy alto grado). Los cinco ítems pertenecientes a cada una de las dimensiones se distribuyen alternos y fluctúan de 5 a 20 puntos. En este sentido, puntuaciones superiores a 12.5 puntos indican un alto grado de estereotipo negativo en dicha dimensión. En el estudio de validación en población española joven y adulta (Menéndez et al., 2016), la escala mostró indicadores de bondad de ajuste satisfactorios (GFI=0,97; RMSR=0,07), una buena consistencia interna $\alpha_{\text {ordinal }}=0.89$ y una estructura unidimensional. En esta investigación, a pesar de que la validación en población menor de 65 años no corroboró la tridimensionalidad de la escala obtenida en la validación original del instrumento (Blanca, Sánchez y Trianes, 2005), se ha considerado necesario analizar los efectos de intervención en las tres subescalas del cuestionario puesto que el objetivo de la investigación es el impacto que puede tener el Programa Acompaña-Té en la reducción de los distintos estereotipos en los participantes del estudio. Es por ello que, para comprobar que las medidas recogidas en el estudio eran fiables, se analizó el nivel de consistencia interna de la escala general y las tres dimensiones (Salud, Motivaciónsocial, Carácter-personalidad respectivamente) a través del coeficiente a de Cronbach (Tabla 2), en ambos momentos temporales (post formación teórica educativa y post formación práctica de contacto intergeneracional), mostrando unos niveles aceptables para el uso del instrumento con fines de investigación, siguiendo los criterios sugeridos por George y Mallery (1995).

Tabla 2. Fiabilidad del CENVE y sus dimensiones en ambos momentos temporales.

\begin{tabular}{ccc}
\hline Evaluación & Escala/dimensión & $\alpha$ Cronbach \\
\hline Fase 1 (Post-teoría) & CENVE_Global & .85 \\
& CENVE_Salud & .56 \\
& CENVE_Mot-soc & .82 \\
Fase 2 (Post-Práctica) & CENVE_Carác-pers & .89 \\
& CENVE_Global & .78 \\
& CENVE_Salud & .65 \\
\end{tabular}




\section{Procedimiento}

La selección de los participantes se realizó durante el mes de febrero de 2018 a través de un procedimiento de selección incidental, tras realizar una entrevista telefónica motivacional para su participación en el Programa Acompaña-Té.

Todos los participantes dieron su consentimiento informado al iniciar el curso y autorizaron el uso anónimo de sus datos, siguiendo los procedimientos éticos de la Declaración de Helsinki y la Ley Orgánica 15/1999, de 13 de diciembre, de Protección de Datos de Carácter Personal. La recogida de datos a través del CENVE para este estudio se realizó en dos momentos temporales, tras finalizar la formación teórica (marzo) y tras realizar la formación práctica contacto intergeneracional (junio), esto es, después de cada componente de la intervención en un intervalo temporal de cuatro meses.

\section{Intervención}

El Programa Acompaña-Té es un curso de voluntariado específico para el acompañamiento de personas ancianas que viven solas que consta de dos módulos de formación: un módulo teórico de 25 horas, que se organiza en seis talleres con formato grupal y metodologías dinámicas de formación, y uno práctico de 25 horas también, en el que se realiza el contacto intergeneracional mediante el acompañamiento de una persona mayor que vive sola durante nueve sesiones en las que se aplica una intervención psicosocial estructurada.

\section{Diseño y análisis de datos}

Se ha realizado un estudio cuasi-experimental con diseño pre-post sin grupo control. Los datos recogidos se registraron y codificaron a través del programa estadístico IBM SPSS Statistics 23. Para el análisis de los resultados se llevaron a cabo análisis descriptivos, mediante medidas de tendencia central y distribución de frecuencias y porcentajes de las variables analizadas. Las diferencias encontradas en los estereotipos negativos hacia la vejez en los dos momentos temporales, tras recibir la formación teórica (Fase 1) y tras realizar la formación práctica de contacto intergeneracional (Fase 2), se analizaron mediante una $t$ de Student para muestras relacionadas, con un nivel de confianza del 95\%. Por último, para estimar el tamaño del efecto de la intervención de acompañamiento sobre las diferencias encontradas, se calculó el estadístico r (Cárdenas y Arancibia, 2014), utilizando los criterios de Cohen para su correcta interpretación: tamaño del efecto pequeño $r \geq 0.10-0.23$; tamaño del efecto medio $r \geq 0.24$ 0.36 ; y tamaño del efecto grande $r \geq 0.37$ (Cohen, 1988).

\section{Resultados}

Análisis descriptivos de los estereotipos negativos hacia el envejecimiento

Las puntuaciones medias en la escala CENVE en los dos momentos temporales: tras la formación teórica $(M=23 ; D T=6.61)$ y tras la formación práctica $(M=21.67 ; D T=6.2)$ se situaron entre baja y muy baja creencia estereotipada. La distribución de puntuaciones por individuo mostró cómo, tras la formación teórica y la práctica de contacto intergeneracional, los participantes revelaron en mayor medida puntuaciones que equivalen a un muy bajo estereotipo.

Después de la formación teórica un $77.78 \%$ de participantes presentó un nivel de estereotipos muy bajo; y tras realizar la formación práctica de contacto intergeneracional el porcentaje fue del 83.33\%. La tendencia a disminuir las creencias estereotipadas se apreció también en cada una de las dimensiones de la escala: Salud, Motivacionalsocial y Carácter-personalidad. Tal y como se refleja en la Tabla 3, tras finalizar la parte práctica de contacto intergeneracional disminuyeron las puntuaciones en cada una de las tres dimensiones respecto a las puntuaciones de la evaluación previa, es decir, tras recibir la formación teórica.

\section{Contrastes de hipótesis y tamaños del efecto}

A través de la prueba t de Student para muestras relacionadas, se comprobó que no existen diferencias estadísticamente significativas en las puntuaciones medias del CENVE en los dos momentos temporales analizados ( $t=1.59$; $p=.13)$, por lo que podemos afirmar que el grado general de creencia estereotipada negativa hacia la vejez (puntuación global del CENVE), no cambió tras realizar la formación práctica de contacto 
Tabla 3. Análisis descriptivo de las puntuaciones en las dimensiones del CENVE.

\begin{tabular}{lcccc}
\hline Dimensiones & $N$ & $M$ & $D T$ & Rango \\
\hline Salud_Post-teoría & 18 & 6.83 & 3.03 & $5-15$ \\
Salud_Post-práctica & 18 & 6.56 & 2.58 & $5-12$ \\
Mot-soc_Post-teoría & 18 & 8.11 & 2.08 & $5-12$ \\
Mot-soc_Post-práctica & 18 & 7.33 & 2.35 & $5-12$ \\
Caráct-pers_Post-teoría & 18 & 6.83 & 2.57 & $5-13$ \\
Caráct-pers_Post-práctica & 18 & 6.56 & 2.28 & $7-16$ \\
\hline
\end{tabular}

intergeneracional. Respecto a las diferencias de medias observadas en las tres dimensiones del CENVE, únicamente fueron estadísticamente significativas las diferencias encontradas en la dimensión Motivacional-social, con un tamaño del efecto medio ( $r=0.50)$, estimándose que un $25 \%$ de los cambios observados en las puntuaciones de dicha dimensión se explica por la participación en la formación práctica de contacto intergeneracional del programa (Tabla 4).

Tabla 4. Contraste de puntuaciones medias

\begin{tabular}{lccc}
\hline Dimensiones & $t_{(17)}$ & $p$ & $r\left(r^{2}\right)$ \\
\hline Salud & 0.61 & .55 & 0.15 \\
& & & $(0.02)$ \\
Motivacional-social & 2.36 & $.03^{*}$ & 0.50 \\
Carácter- & & & $(0.25)$ \\
personalidad & 0.86 & .40 & 0.21 \\
${ }^{*} p<.05$ & & & $(0.04)$ \\
\hline
\end{tabular}

\section{Discusión}

Con el presente estudio se ha comprobado cómo aquéllos que realizaron un aprendizaje experiencial, realizando una intervención estructurada de acompañamiento a domicilio de una persona mayor que vivía sola durante 25 horas, consiguieron disminuir los estereotipos negativos relacionados con los aspectos motivacionales y sociales que tenían sobre las personas mayores tras finalizar la formación teórica del curso de voluntariado del Programa Acompaña-Té. Esto es, a través del contacto intergeneracional que tuvieron durante el acompañamiento cambió la imagen que los participantes tenían sobre los intereses vitales, conductas de pasividad e inactividad física y social y alto grado de aislamiento de las personas mayores.

En el estudio anterior sobre la efectividad para reducir los estereotipos negativos de la formación teórica del Programa referido (Brotons et al., en prensa), únicamente se encontraron cambios en las dimensiones Salud y Carácter-personalidad de los estereotipos que tenían los participantes. Los mismos resultados encontraron Elliott y Rubio (2017), pero en este caso, tras implementar un programa de intervención que promovía el contacto intergeneracional durante nueve sesiones; lo cual es llamativo, puesto que, a pesar de que Elliott y Rubio utilizan un programa de contacto intergeneracional de nueve sesiones, que coincide con la estructura del que se ha utilizado en este estudio, sus hallazgos difieren de los que se han encontrado aquí. Estas diferencias pueden deberse a las características del contacto intergeneracional que se promueve en cada programa de intervención empleado en los estudios, puesto que las nueve sesiones de contacto intergeneracional que se proponen en el Programa Acompaña-Té estaban orientadas hacia el conocimiento y la estimulación de las motivaciones personales y la participación social de las personas mayores. De esta forma, se facilita que los participantes superen la imagen estereotipada de la vejez basada en la edad y las pérdidas y, a su vez, se desarrolla una imagen particularmente más positiva de la vejez en las tres dimensiones que evalúa el CENVE. 
Respecto a la eficacia del tipo de intervención, los resultados encontrados siguen la propuesta que Levy (2016) plantea sobre los programas combinados en el Modelo PEACE. Aunque los resultados de los dos estudios experimentales que realizaron para comprobar el modelo no apoyaron esta hipótesis (Lytle y Levy, 2017), es preciso señalar que en dichos estudios se consideró como 'contacto intergeneracional' el hecho de transmitir información sobre terceras personas (familiares, amigos/as, etc.) que tenían contactos intergeneracionales positivos. Es decir, no se puso a prueba la efectividad del contacto intergeneracional real, sino la información sobre este tipo de relaciones. En el presente estudio, en cambio, el componente de contacto intergeneracional que hemos incorporado no ha sido informacional, sino que ha consistido en una intervención de contacto intergeneracional directa e individualizada $y$, además, los participantes habían recibido previamente una formación teórica específica y más extensa que la utilizada en los estudios de Lytle y Levy (2017). Estas diferencias señaladas a la hora de aplicar los componentes de intervención podrían explicar los cambios que se han encontrado en este estudio entre la formación teórica (intervención educativa sobre el envejecimiento) y la formación práctica (intervención de contacto intergeneracional).

Por todo lo expuesto se puede afirmar que, a través de la práctica de contacto intergeneracional, sí se pueden modificar los estereotipos edadistas, tal y como defiende el modelo PEACE pues, tal y como se ha demostrado, los participantes de este estudio, tras haber vivido la experiencia de interactuar con una persona mayor, consiguieron disminuir sus estereotipos negativos relacionados con los aspectos motivacionales y sociales de las personas acompañadas; estereotipos que no hubiesen disminuido si hubiesen recibido únicamente la formación teórica del programa.

El presente estudio no está exento de limitaciones como es el pequeño tamaño muestral que compromete la potencia de los contrastes de hipótesis. Asimismo, la selección incidental de la muestra y la ausencia de un grupo control que permita contrastar el efecto de la intervención y no intervención sobre los estereotipos en ambos grupos son aspectos que pueden ser mejorados en futuras investigaciones. A pesar de ello, los hallazgos presentados en este trabajo son novedosos y pioneros en nuestro país. Suponen una evidencia en favor de la implementación de programas intergeneracionales combinados en contextos universitarios para modificar estereotipos negativos hacia la vejez $\mathrm{y}$, por consiguiente, para el desarrollo de estrategias de intervención económicas en salud pública que repercutan en un verdadero cambio social para eliminar el edadismo. Para seguir aportando más luz en esta línea, en futuras investigaciones sería conveniente realizar este tipo de estudios con otras poblaciones, no únicamente universitaria, donde los participantes se dividan en tres grupos de intervención: teoría, interacción intergeneracional y mixto.

\section{Conclusiones}

Con este estudio se ha comprobado empíricamente el efecto positivo que tiene un aprendizaje experiencial a partir del contacto intergeneracional, sobre los efectos ya conocidos de una formación teórica sobre el envejecimiento, para eliminar los estereotipos negativos hacia la vejez en estudiantes universitarios. Este hecho evidencia el potencial del aprendizaje experiencial en la educación universitaria y, concretamente, de los programas combinados (formación teórica sobre envejecimiento junto con la experiencia personal directa de contacto intergeneracional) para superar estereotipos negativos hacia la vejez, promover la solidaridad intergeneracional y fomentar una visión positiva hacia el proceso de envejecimiento.

Para seguir aportando más evidencias en este ámbito de investigación, es necesario desarrollar ensayos controlados aleatorizados en contextos educativos que permitan analizar la efectividad de este modelo de intervención para eliminar los estereotipos negativos hacia la vejez. Las evidencias encontradas hasta ahora, así como las referidas en el presente, pueden servir como modelo de intervención contra el edadismo en contextos educativos de distinta índole, ya que son fácilmente replicables y adaptables a otros entornos y, además, la implementación de estas intervenciones supondrá una oportunidad perfecta para poder analizar la efectividad en contextos reales, asegurando así la validez ecológica de los hallazgos encontrados. 


\section{Referencias}

Abellán-García, A., Aceituno-Nieto, P., PérezDíaz, J., Ramiro-Fariñas, D., Ayala-García, A. y Pujol-Rodríguez, R. (2019). 'Un perfil de las personas mayores en España, 2019. Indicadores estadísticos básicos'. Madrid, Informes Envejecimiento en red $n^{\circ} 22$, 38p. Recuperado de http://envejecimiento. csic.es/documentos/documentos/enredindicadoresbasicos2019.pd

Blanca, M. J., Sánchez, C. y Trianes, M. V. (2005). Cuestionario de la evaluación de estereotipos negativos haciala vejez. Revista Multidisciplinar de Gerontología. 15(4), 212-220.

Blanco-Molina, M. y Pinazo-Hernandis, S. (2016). Información y exposición indirecta para reducir estereotipos hacia el envejecimiento. International Journal of Developmental and Educational Psychology, 1(2), 367-379. doi: 10.17060/ijodaep.2016.n2.v1.707

Borrella, M. S. (2013). Programa educativo para fomentar actitudes positivas en adolescentes hacia los mayores (Tesis Doctoral), Universidad de Extremadura, España. Recuperado de http://hdl.handle.net/10662/798

Brotons, P., Lorente, R. y Sitges, E. (En prensa). Efectos del Programa ACOMPAÑA-TÉ sobre los estereotipos hacia la vejez en universitarios. Revista de Psicología de la Salud.

Butler, R. (1969). Age-ism: Another form of bigotry. The Gerontologist, 9, 243-246. doi: https:// doi.org/10.1093/geront/9.4_Part_1.243

Carbajo-Vélez, M. C. (2009). Mitos y estereotipos sobre la vejez. Propuesta de una concepción realista y tolerante. ENSAYOS, Revista de la Facultad de Educación de Albacete, 24, 8796. Recuperado de https://dialnet.unirioja.es/ servlet/articulo?codigo $=3282988$

del Campo, S. D. A. y Maestro, R. L. (2014). Análisis crítico del discurso publicitario institucional/comercial sobre las personas mayores en España. Comunicar: Revista científica iberoamericana de comunicación y educación, 42, 189-197. doi: https://doi. org/10.3916/C42-2014-19
Elliott, P. V. y Rubio, L. (2017). Cambios en los estereotipos sobre la vejez de estudiantes tras su participación en un proyecto intergeneracional. International Journal of Developmental and Educational Psychology. Revista INFAD de Psicología, 1(2), 61-68. doi: http://dx.doi.org/10.17060/ijodaep.2017. n2.v1.1108

George D. y Mallery. P. (1995). SPSS/PC step by step: A simple guide and reference. Belmont, CA: Wadsworth Publishing Company.

Gonçalves, M., Hatton-Yeo, A. y Farcas, D. (2016). Overcoming ageism through a new intergenerational learning paradigm: challenges and findings. Quality in Ageing and Older Adults, 17(4), 239-245. doi: 10.1108/ QAOA-04-2015-0016

Gutiérrez, M. y Mayordomo, T. (2019). Edadismo en la escuela. ¿Tienen estereotipos sobre la vejez los futuros docentes? Revista Educación, 43(2), 19-19. doi: 10.15517/ REVEDU.V43I2.32951

Levy, S. R. (2016). Toward reducing ageism: PEACE (positive education about aging and contact experiences) model. The Gerontologist, 58(2), 226-232. doi: https://doi.org/10.1093/geront/ gnw116

Lytle, A. y Levy, S. R. (2017). Reducing ageism: Education about aging and extended contact with older adults. The Gerontologist, 59(3), 580-588. doi: 10.1093/geront/gnx177

Lytle, A. (2016). A comparison of two theoretical approaches to addressing ageism: Education and extended contact (doctoral dissertation. Stony Brook University, Stony Brook, NY. Recuperado de http://hdl.handle. net/11401/76794

Menéndez, S., Cuevas-Toro, A. M., PérezPadilla, J. y Lorence, B. (2016). Evaluación de los estereotipos negativos hacia la vejez en jóvenes y adultos. Revista Española de Geriatría y Gerontología, 51(6), 323-328. doi: 10.1016/j.regg.2015.12.003 
Moreno, A. (2010). Viejismo (ageism). Percepciones de la población acerca de la tercera edad: estereotipos, actitudes e implicaciones sociales. Poiésis, 10(19). doi: https://doi.org/10.21501/16920945.101

Organización Mundial de la Salud (2015). Informe Mundial sobre el Envejecimiento y la Salud. OMS: Estados Unidos de América. Recuperado 12/6/2019 en: https:// apps.who.int/iris/bitstream/handle/10 $665 / 186466 / 9789240694873$. spapdf;jsessionid=957A85295B7B7B3 1C41C161C97E887A7? sequence $=1$

Pinazo-Hernandis, S. y Pinazo-Clapés, C. (2018). Literaturay transgeneracionalidad. Un proyecto intergeneracional de apadrinamiento lector en ámbito residencial. Ocnos: Revista de estudios sobre lectura, 17(3), 42-54. doi: https://doi. org/10.18239/ocnos_2018.17.3.1799

Rello, C., F., Bravo, M. D. L. y Plata, R. M. M. (2018). Estereotipos sobre la edad y el envejecimiento en estudiantes y profesionales de Ciencias de la Salud. Prisma Social: revista de investigación social, 21, 108-122. Recuperado de https://dialnet.unirioja.es/ servlet/articulo?codigo $=6521445$

Ribera, J. M., Bustillos, A., Guerra, A. I., Huici, C. y Fernández-Ballesteros, R. (2016). ¿Se discrimina a los mayores en función de su edad? Visión del profesional. Revista Española de Geriatría y Gerontología, 51(5), 270-275. doi: 10.1016/j.regg.2016.03.005

Sanhueza Chamorro, J. (2014). Imágenes sobre la vejez en jóvenes estudiantes universitarios según área del conocimiento: Un estudio exploratorio. Acciones $E$ Investigaciones Sociales, 34, 105-124. doi: 10.26754/ojs_ais/ ais. 201434913

Sarabia C. M. y Castanedo, C. (2015). Modificación de estereotipos negativos en la vejez en estudiantes de enfermería. Gerokomos, 26(1), 10-12. Recuperado de http://scielo.isciii.es/ pdf/geroko/v26n1/original2.pdf
Snyder, C. S., Wesley, S. C., Lin, M. B. y May, J. D. (2008). Bridging the gap: Gerontology and social work education. Gerontology \& Geriatrics Education, 28(4), 1-21. doi: 10.1080/02701960801962864

Zabalza, M. A. (2013). El practicum como situación de aprendizaje. En M.A. Zabalza (2013), El Practicum y las Prácticas en Empresas (103134). Madrid, España: Narcea. 